\title{
COMPACT TRANSFORMATIONS AND THE $k$-TOPOLOGY IN HILBERT SPACE ${ }^{1}$
}

\author{
RALPH A. RAIMI
}

1. Introduction. We shall be concerned only with Hilbert spaces, though many of the notions involved can be extended to-and indeed sometimes originally appeared in-a wider context. By a Hilbert space $H$ will be meant an inner product space of arbitrary dimension, which is moreover complete. The $k$-topology for $H$, originally defined in $[1],{ }^{2}$ is generated from a basis set of neighborhoods of the identity $\theta,\{V(\theta)\}$, obtained in the following way: If $K$ is any compact subset of $H$, let $V(\theta)=\{x \in H||(x, y) \mid \leqq 1$ for all $y \in K\}$. The interest of the $k$-topology lies in the fact that it is the strongest locally convex topology for $H$ which coincides with the weak topology on all spheres [2].

The purpose of this paper is to introduce an equivalent method of defining this topology, the equivalence to be proved via a lemma concerning compact transformations on $H$ to $H$.

2. Compact transformations and sets in $H$. Lemma 1 is probably well known, being an explicit form of some more general theorems concerning pointwise and uniform convergence of sequences of continuous mappings of a compact set.

Lemma 1. Let $H$ be a Hilbert space with a denumerable orthonormal base $\left(e_{1}, e_{2}, \cdots, e_{n}, \cdots\right)$ in terms of which every element $x \in H$ has the unique expansion $x=\sum_{i=1}^{\infty} a_{i}(x) e_{i}$. Let $K$ be a closed bounded subset of $H$. Then $K$ is compact if and only if the following criterion holds: For every real $\epsilon>0$, there exists an integer $N(\epsilon)$ such that $\left\|\sum_{i=N+1}^{\infty} a_{i}(x) e_{i}\right\|$ $<\epsilon$ for all $x$ in $K$.

A linear transformation $T: H \rightarrow H$ is called compact if $\mathrm{Cl}(T(S))$, the closure of $T(S)$, is compact, where $S$ is the unit ball $\{x \in H \mid\|x\|$ $\leqq 1\}$.

Lemma 2. Let $H$ be any Hilbert space, and $K$ any compact subset. Then there exists a compact linear transformation $T$ such that $T(S) \supset K$. 1954.

Presented to the Society, October 30,1954; received by the editors October 6 ,

1 This paper is a short extract from a thesis presented to the University of Michigan for the degree of $\mathrm{Ph}$.D. The work was supported in part by the Office of Naval Research, Contract Nonr-330(00).

2 Numbers in brackets refer to the bibliography at the end of the paper. 
Proof. We may assume without loss of generality that (1) $K$ is closed, convex, symmetric, and (2) that $K \subset S$. By symmetric is meant that if $x \in K$, and if $a$ is a scalar such that $|a|=1$, then $a x \in K$. (1) is possible because if $K$ is extended to its least convex, symmetric, closed hull, the result is still compact. Proving the lemma for this hull proves it a fortiori for $K$. (2) follows from the fact that a scalar multiple of a compact transformation is again compact.

Let $a_{1}=\sup \{\|x\| \mid x \in K\}$. Since $K$ is compact, and $\|x\|$ is a continuous function on $K$, there is a vector $y_{1} \in K$ at which the norm $a_{1}$ is taken on. Set $e_{1}=y_{1} / a_{1}$. Then $e_{1}$ is the first element of an orthonormal sequence constructed inductively as follows: Denote by $V_{n}$ the linear extension of $\left\{e_{1} \cup e_{2} \cup \cdots \cup e_{n}\right\}$, and by $V_{n}^{\perp}$ the orthogonal complement of $V_{n}$ in $H$. For any element $x \in K$, there is an unique decomposition $x=x^{n}+u^{n}$, with $x^{n} \in V_{n}$ and $u^{n} \in V_{n}^{\perp}$. Then set $a_{n+1}$ $=\sup \left\{\left\|u^{n}\right\| \mid x \in K, x=x^{n}+u^{n}\right\}$. Since $u^{n}$ and hence $\left\|u^{n}\right\|$ is a continuous function on the compact $K$, there exists a vector $x=x^{n}+u^{n}$ at which this supremum is taken on. Let $y_{n+1}=u^{n}$ for this $x$ and set $e_{n+1}$ $=y_{n+1} / a_{n+1}$, where $a_{n+1}$ is the supremum in question. Clearly the vectors $y_{n}$, and hence the unit vectors $e_{n}$, form an orthogonal set, with $y_{n+1}$ and hence $e_{n+1}$ orthogonal to $V_{n}$. It is also clear from the construction that $a_{n+1} \leqq a_{n}$ for all $n$. Moreover, $\lim _{n} a_{n}=0$, for if this were not the case, there is some $\delta>0$ such that $a_{n}>\delta$ for all $n$. But this is to say that in the construction, if $x_{n}$ is the sequence in $K$ at which the successive maxima are taken on, and if we represent $x_{n}=x^{n}+u^{n}$ as above, then $\left\|u^{n}\right\|>\delta$ for all $n$. Then for $n>m,\left\|x_{m}-x_{n}\right\|=\| x^{m}-x^{n}$ $+u^{m}-u^{n}\|\geqq\| u^{n} \|>\delta$, because the sum of the first three terms is in $V_{n}$ while the fourth is orthogonal to $V_{n}$. Hence the sequence $\left\{x_{n}\right\}$ has no point of accumulation, denying the compactness of $K$.

Let $\mathfrak{F C}$ be the closure of $U_{1}^{\infty} V_{n}$. $\mathcal{F C}$ is complete and separable, i.e. it has a countable orthonormal base, the set $\left\{e_{n}\right\}$ in fact. Further, $\mathfrak{H} \supset K$. For, if $y \in K, y=y^{n}+u^{n}$, with $y^{n} \in V_{n} \subset \mathcal{F}$, and $\left\|u^{n}\right\| \leqq a_{n+1}$ which becomes arbitrarily small with increasing $n$. Hence $y$ is arbitrarily close to $\cup_{1}^{\infty} V_{n}$, the closure of which is $\mathcal{H}$. Thus, for any $x \in K, x=\sum_{1}^{\infty} x_{i} e_{i}$, and $\left\|\sum_{n}^{\infty} x_{i} e_{i}\right\|=\left(\sum_{n}^{\infty}\left|x_{i}\right|^{2}\right)^{1 / 2} \leqq a_{n}$ for all $n$. We also observe, by assumption (2) of the opening of this proof, that $a_{1} \leqq 1$.

Now let $b_{n}=\left(2 a_{n}\right)^{1 / 2}$. Clearly $b_{n} \geqq\left(a_{n}\right)^{1 / 2} \geqq a_{n}$, since $a_{n} \leqq 1$. Also, $b_{n} \geqq b_{n+1}$ for all $n$, and $\lim _{n} b_{n}=0$. We construct $T$ as follows: Let $T e_{i}=b_{i} e_{i}$ for all $i$, and extend $T$ by linearity and continuity to all of F. For $x \in \mathcal{F}^{\perp}$, define $T x=\theta$, and again $T$ may be extended by linearity, this time to all of $H$.

To show $T$ is a compact transformation, it will suffice to apply 
Lemma 1 to the set $\mathrm{Cl}(T(S))$. Let $\epsilon>0$ be given, and choose $N$ such that $b_{N}<\epsilon / 2$. Now if $y=\sum_{1}^{\infty} y_{i} e_{i} \in \operatorname{Cl}(T(S))$, there exists some $x=\sum_{1}^{\infty} x_{i} e_{i} \in T(S)$ such that $\|x-y\|<\epsilon / 2$. Further, there exists some $z=\sum_{1}^{\infty} z_{i} e_{i} \in S$ such that $T z=x$, i.e. $\sum_{1}^{\infty} z_{i} b_{i} e_{i}=\sum_{1}^{\infty} x_{i} e_{i}$. Then $\left\|\sum_{N}^{\infty} y_{i} e_{i}\right\| \leqq\left\|\sum_{N}^{\infty}\left(y_{i}-x_{i}\right) e_{i}+\sum_{N}^{\infty} x_{i} e_{i}\right\| \leqq\|y-x\|+\left\|\sum_{N}^{\infty} \quad b_{i} z_{i} e_{i}\right\|$ $<\epsilon / 2+b_{N}\|z\|<\epsilon$. Thus the criterion of Lemma 1 is fulfilled.

It only remains to be shown that $T(S) \supset K$. To this end, let $x=\sum_{1}^{\infty} x_{i} e_{i} \in K$. Then if $z=\sum_{1}^{\infty}\left(x_{i} / b_{i}\right) e_{i}, T z=x$. To show $z \in S$ completes the proof. We shall show $\|z\|^{2} \leqq 1$ by showing that

$$
\sum_{1}^{n}\left(\left|x_{i}\right|^{2} / b_{i}^{2}\right) \leqq 1
$$

for all $n$. If we set $R_{n}=\sum_{n}^{\infty}\left|x_{i}\right|^{2}$, and observe that $R_{n} \leqq a_{n}^{2}$, then

$$
\begin{aligned}
\sum_{1}^{n}\left(\left|x_{i}\right|^{2} / b_{i}^{2}\right) & =\sum_{1}^{n} \frac{R_{i}-R_{i+1}}{2 a_{i}} \\
& =\frac{1}{2}\left[\sum_{0}^{n-1} \frac{R_{i+1}}{a_{i+1}}-\sum_{1}^{n} \frac{R_{i+1}}{a_{i}}\right] \\
& =\frac{1}{2}\left[\left(\frac{R_{1}}{a_{1}}-\frac{R_{n+1}}{a_{n}}\right)+\sum_{1}^{n-1} R_{i+1}\left(\frac{1}{a_{i+1}}-\frac{1}{a_{i}}\right)\right] \\
& \leqq \frac{1}{2}\left[a_{1}+\sum_{1}^{n-1} a_{i+1}^{2}\left(\frac{a_{i}-a_{i+1}}{a_{i+1} a_{i}}\right)\right] \\
& =\frac{1}{2}\left[a_{1}+\sum_{1}^{n-1} \frac{a_{i+1}}{a_{i}}\left(a_{i}-a_{i+1}\right)\right] \\
& \leqq \frac{1}{2}\left[a_{1}+\sum_{1}^{n-1}\left(a_{i}-a_{i+1}\right)\right] \\
& =\frac{1}{2}\left[a_{1}+a_{1}-a_{n}\right] \\
& \leqq a_{1} \leqq 1 .
\end{aligned}
$$

3. Alternate definition of the $k$-topology. Let $C(H, H)$ denote the class of all compact linear transformations, and let the c-topology be defined as follows: The typical neighborhood of $\theta, V(\theta)=\{x \in H \mid$ $\left.\left\|T_{i} x\right\| \leqq 1, T_{i} \in C(H, H), i=1,2, \cdots, n\right\}$. A basis of $c$-neighborhoods of $\theta$ is obtained by letting the finite sets $\left\{T_{i}\right\}$ run through the class of all finite subsets of $C(H, H)$. That a locally convex topology is thus produced for $H$ is easily verified directly; indeed the $c$-topology is nothing but the point-open topology on $H$, when $H$ is regarded as a space of functions from $C(H, H)$ to $H$, under the rule $x: T \rightarrow T x$. 
Theorem. The topologies $c$ and $k$ are identical.

Proof. Let $V(\theta)$ be any $k$-neighborhood: $V(\theta)=\{x \in H||(x, y) \mid \leqq 1$ for all $y \in K, K$ compact $\}$. Let $T \in C(H, H)$ be chosen (according to Lemma 1) so that $T(S) \supset K$, and let $T^{\prime}$ be the adjoint of $T$. As is well known, $T^{\prime} \in C(H, H)$ also. If $W$ is the $c$-neighborhood $\{x \in H \mid$ $\left.\left\|T^{\prime} x\right\| \leqq 1\right\}$, then $W \subset V$, for let $x \in W$. Then $\left\|T^{\prime} x\right\| \leqq 1$, and if $u \in S$, $\left|\left(u, T^{\prime} x\right)\right| \leqq 1$. Thus $|(T u, x)| \leqq 1$ for all $u \in S$, and a fortiori, $|(y, x)| \leqq 1$ for all $y \in K$. Conversely, let $W(\theta)$ be a $c$-neighborhood: $W=\left\{x \in H \mid\left\|T_{i} x\right\| \leqq 1, T_{i} \in C(H, H), i=1,2, \cdots, n\right\}$. Then for all $u \in S,\left|\left(u, T_{i} x\right)\right| \leqq 1$, and $\left|\left(T_{i}^{\prime} u, x\right)\right| \leqq 1$, for each $i$. The $T_{i}^{\prime}$ are all in $C(H, H)$. Let $K=\bigcup_{i=1}^{n} \mathrm{Cl}\left(T_{i}^{\prime}(S)\right)$. Then the $k$-neighborhood $V(\theta)=\{x \in H||(y, x) \mid \leqq 1$ for all $y \in K\}$ clearly satisfies $V(\theta) \subset W(\theta)$. Q.E.D.

The definitions for the $k$ - and $c$-topologies can be generalized considerably, but even for complete normed spaces having a Schauder base it is not possible to prove Lemma 2 by the device used above. However, a statement somewhat weaker than Lemma 2 would suffice: that given a compact set $K$, there exist a finite number of compact transformations $T_{i}$ such that the union of $T_{i}(S)$ contain $K$.

\section{BIBLIOGRAPHY}

1. R. Arens, Duality in linear spaces, Duke Math. J. vol. 14 (1947) pp. 787-794.

2. J. Dieudonne, Natural homomorphisms in Banach spaces, Proc. Amer. Math. Soc. vol. 1 (1950) pp. 54-59.

UNIVERSITY OF MiChIGAN 